\title{
A mission to test the Pioneer anomaly: estimating the main systematic effects
}

\author{
O. Bertolami *, J. Páramos ${ }^{\dagger}$ \\ Instituto Superior Técnico, Departamento de Física, \\ Av. Rovisco Pais 1, 1049-001 Lisboa, Portugal and \\ E-mail addresses: orfeu@cosmos.ist.utl.pt, x_jorge@fisica.ist.utl.pt
}

(Dated: April 19, 2018)

\begin{abstract}
We estimate the main systematic effects relevant in a mission to test and characterize the Pioneer anomaly through the flight formation concept, by launching probing spheres from a mother spacecraft and tracking their motion via laser ranging.
\end{abstract}

\section{INTRODUCTION}

Analyses of radiometric data of the Pioneer 10 and 11 spacecraft do suggest the existence of an anomalous acceleration on the two spacecraft, inbound to the Sun and with a (constant) magnitude of $a_{P} \simeq(8.5 \pm 1.3) \times 10^{-10} \mathrm{~m} / \mathrm{s}^{2}$. Extensive attempts carried out so far to explain this phenomena as a result of an estimate of the main systematic effects accounting for thermal and mechanical effects, as well as errors in the tracking algorithms used, have shown to be unsuccessful [1], despite claims otherwise [2]. Also, another analysis has shown that a secular trend in the anomaly may be found, with a time constant larger than 50 years allowed: this still leaves room for thermal radiation to account for the Pioneer anomaly (given the $\sim 88$ years half-life of the plutonium source in the radio-thermal generators, which should be somewhat lowered due to degradation of the thermal coupling), and is undergoing further studies by groups within the Pioneer collaboration team [3]. The two Pioneer spacecraft follow approximate opposite hyperbolic trajectories away from the Solar System but, despite that, the same anomaly was found. This prompts for an intriguing question: what is the fundamental, and possibly new, physics behind this anomaly?

To answer this, many proposals have been advanced, as summarized in Ref. [4]. However, before the possibility for new physics is seriously addressed, an unequivocal description of the anomaly, as given by already available data, should be given. In particular, secular and spatial trends should be carefully modeled, both from a statistical point of view and as possible thermal and engineering causes of the anomalous acceleration. Furthermore, the direction of the acceleration vector must be clearly characterized. Indeed, the distances involved in the already conducted Doppler analysis do not allow for an angular discrimination between the several candidates, each remarking a different origin for the anomaly: a line of action pointing towards the Sun would indicate an effect of gravitational origin (since solar pressure is manifestly too low to account for the effect), while a Earth-bound anomaly would indicate either a modified Doppler effect (and hence, new physics affecting light propagation and causing an effective blue shift), or an incorrect modeling of Doppler data, possibly due to Deep Space Network and software clock drifts, incorrect ephemerides estimates, mismodeled Earth orientation parameters, etc; the anomaly may also point along the spin axis of the spacecraft, indicating that onboard, underestimated systematic effects are responsible for it; an anomaly along the velocity vector would hint at some sort of drag effect, possibly related to dark matter or dust distribution (although these are currently known to a good accuracy, and yield much lower effects), or to a modification of geodetical motion also hinting at a modification or extension of General Relativity.

Clearly, it is difficult to correctly assign a definitive origin to the observables of interest: the direction, magnitude, spatial and secular variation of the anomaly. This further enforces the need to carefully account for all known effects, despite the added difficulty of dealing with data that is almost thirty years old! For sure, rather complex and subtle model-dependent computations must be carried out - preferably starting with different assumptions and hopefully getting the same final answer for the enigma of the Pioneer anomaly.

\section{ACCELERATION BUDGET}

In order to test the Pioneer anomaly, one must fully characterize its features by removing the uncertainties due to systematics and other biases, and ascertain the magnitude and direction of the anomalous acceleration. Thus, we begin by listing the various components that may affect an extended object, a spacecraft, traveling through the solar system:

† Also at Centro de Física dos Plasmas, Instituto Superior Técnico, Lisbon. 


$$
\mathbf{F}=\mathbf{F}_{\text {Grav }}+\mathbf{F}_{\text {Pres }}+\mathbf{F}_{\text {Drag }}+\mathbf{F}_{L}+\mathbf{F}_{\text {Syst }}+\mathbf{F}_{\text {Unk }}
$$

where the underscripts indicate the origin of the various components. The gravitational force $\mathbf{F}_{\text {Grav }}$ is given by the sum of the Newtonian forces corresponding to all the major bodies in the solar system, yielding about $a_{G r a v}=$ $\Sigma_{i} G M_{i} m / r_{i}^{2}=6 \times 10^{-3} \mathrm{~m} / \mathrm{s}^{2}$ at a distance from the Sun of $1 \mathrm{AU}$. The solar pressure $\mathbf{F}_{\text {Pres }}$ is divided into a radiation component, given by $K f_{\odot} A \cos \theta(r) / c r^{2}$, where $c$ is the speed of light, $K$ is the effective absorption/reflection coefficient (of order unity), $f_{\odot}$ is the effective-temperature "solar radiation constant" - approximately $1.4 \mathrm{~kW} / \mathrm{m}^{2}(A U)^{2}$ at $r=1 A U-, A$ is the projected area to the solar vector, $\theta$ is the angle between the spacecraft antenna's axis and the direction of the Sun, and $r$ is the distance from the Sun; for a cross-sectional area of the order of $10 \mathrm{~m}^{2}$, this yields approximately $a_{\text {Pres }}=10^{-8}-10^{-7} \mathrm{~m} / \mathrm{s}^{2}$ (depending on assumptions) at $r=1 \mathrm{AU}$, and falls below the reported magnitude of the Pioneer anomaly at $r \approx 11 A U$ (for the upper bound, or a much closer $r \approx 3.5 A U$ for the lower bound) - well within the flight envelope of a typical deep space mission. The solar wind component is given by a similar expression, with $f_{\odot}$ substituted by $m_{p} v^{3} n$, where $n \approx 5 \mathrm{~cm}^{-3}$ is the proton density at $1 A U$ and $v \approx 400 \mathrm{~km} / \mathrm{s}$ is the solar wind speed, this yields a value of the order $a_{\text {wind }}=10^{-13}-10^{-12} \mathrm{~m} / \mathrm{s}^{2}$. The drag force due to interplanetary dust is given by $-k A \rho(r) v(r)^{2} \hat{\mathbf{v}}$, where $k$ is a characteristic constant of order unity, $A$ is the cross-section, $\rho \approx 10^{-24} \mathrm{~g} / \mathrm{cm}^{3}$ is the interplanetary medium density and $v \approx 400 \mathrm{~km} / \mathrm{s}$ is the body's velocity; this yield approximately $a_{D r a g}=10^{-12}-10^{-11} \mathrm{~m} / \mathrm{s}^{2}$. The electromagnetic Lorentz force, related to local magnetic field $\mathbf{B}$, the probe's charge $q$ and velocity $\mathbf{v}$ by $\mathbf{F}_{L}=q \mathbf{v} \times \mathbf{B}$; although the accumulated charge in the spacecraft and the magnetic field may vary widely, a upper bound of $a_{L} \sim 10^{-12} \mathrm{~m} / \mathrm{s}^{2}$ is a typical value [5]. Finally, the systematic component accounts for possible gas leakage, anisotropic heat emission and reflectance, antenna recoil and other effects; and, finally, we consider a component of unknown origin.

The analysis of the relevant systematic components can be simplified by comparing the precision of acceleration measurements with the order of magnitude of the above components: when the former is higher than the latter, one can safely disregard them from the overall picture. In the following, we assume that the low systematics intended for a dedicated probe allow for a Doppler tracking accuracy of order $10^{-11} \mathrm{~m} / \mathrm{s}^{2}$, and a direct measurement through internal accelerometer of order $10^{-12} \mathrm{~m} / \mathrm{s}^{2}$. The former is one order of magnitude lower than the accuracy available for the Pioneer data, and the latter corresponds to state of the art low-frequency accelerometers.

An obvious statement concerning any dedicated mission to test the Pioneer anomaly is that it should be more robust than these scientific probes, that is, that the preceding components for the Doppler and acceleration measurements should be of smaller magnitude than those affecting the Pioneer spacecraft. Hence, from Table II of Ref. [1], we get the following upper limits for these components:

As stated in Ref. [1], one can safely assume that the anomalous acceleration is not due to electromagnetic forces, nor to solar radiation and solar wind pressures. Also, it is argued that the drag due to the surrounding environment is not enough to account for the anomaly; regarding this, one may ask what should be the medium's density, so that a $v^{2}$ dependent drag force would account for the anomaly: a simple calculation shows that this should be of order $10^{-19} \mathrm{~g} / \mathrm{cm}^{3}$; for comparison, the density of interplanetary dust, arising from hot-wind plasma [6], is lesser than $10^{-24} \mathrm{~g} / \mathrm{cm}^{3}$; the density of interstellar dust (directly measured by the Ulysses spacecraft) is even smaller, at about $3 \times 10^{-26} \mathrm{~g} / \mathrm{cm}^{3}$.

Also, an under-estimated mass of the yet unexplored Kuiper belt could possibly account for the anomalous acceleration. However, it has been shown that this would require an abnormally high mass for this extended object, about two order of magnitude higher than the commonly accepted value of $M_{\text {Kuiper }}=0.3 M_{\text {Earth }}[1,7,8]$.

The above discussion supports the acknowledged status of the Pioneer anomaly, as currently stated by the Pioneer anomaly collaboration: the origin of the anomaly can be due to systematics, such as underestimated thermal effects, or to new physics. Clearly, the presented error budget shows that the systematics errors cannot account for the anomaly; however, this figure was obtained resorting to the available data concerning RTG power decay and emissivity, radio antenna emissions, Helium leak in the RTGs, amongst other competing effects (see Ref. [1] for a full discussion). In principle, it is conceivable that a yet unaccounted systematic effect can explain the anomaly. For this reason, it is not sufficient to design a dedicated probe in such a way as to minimize known systematic effects, namely through a careful thermal modeling and symmetrical geometry; indeed, a key feature of the proposed dedicated probe [3] is that it allows for a direct assessment of these systematic perturbations.

As proposed in Ref. [3], this is attained thanks to the direct measurement of the distance between the primary craft and a small passive sphere - circa $10 \mathrm{~cm}$ diameter and with no attitude or stabilization capability. This could be attained either by endowing the latter with a low power transponder, or by covering it with cornercube retroreflectors, allowing for laser-ranging from the primary craft - these devices reflect any incoming light ray in the incoming direction, eliminating the need for a perfect alignment between the laser sight and the passive sphere. Asides from design simplicity, a small sphere allows for a better characterization of its surface and thermal properties, and also yields smaller and more easily modeled temperature gradients; also, the lack of attitude control allows 
TABLE I: Error budget for the Pioneer 10 and 11, taken from Ref. [1].

\begin{tabular}{ll} 
Item Description of error budget constituents & Bias $\begin{array}{l}\text { Uncertainty } \\
10^{-8} \mathrm{~cm} / \mathrm{s}^{2} 10^{-8} \mathrm{~cm} / \mathrm{s}^{2}\end{array}$ \\
\hline
\end{tabular}

1 Systematics generated external to the spacecraft:
a) Solar radiation pressure and mass
$+0.03$
$\pm 0.01$
b) Solar wind
$\pm<10^{-5}$
c) Solar corona
d) Electro-magnetic Lorentz forces
e) Influence of the Kuiper belt's gravity
$\pm 0.02$
f) Influence of the Earth orientation
$\pm<10^{-4}$
$\pm 0.03$
g) Mechanical and phase stability of DSN antennae
$\pm 0.001$
h) Phase stability and clocks
$\pm<0.001$
i) DSN station location
$\pm<0.001$
j) Troposphere and ionosphere
$\pm<10^{-5}$
$\pm<0.001$

2 On-board generated systematics:
a) Radio beam reaction force
$+1.10 \quad \pm 0.11$
b) RTG heat reflected off the craft
$-0.55 \quad \pm 0.55$
c) Differential emissivity of the RTGs
$\pm 0.85$
d) Non-isotropic radiative cooling of the spacecraft
$\pm 0.48$
e) Expelled Helium produced within the RTGs
$+0.15$
$\pm 0.16$
f) Gas leakage
$\pm 0.56$
g) Variation between spacecraft determinations
$+0.17$
$\pm 0.17$

3 Computational systematics:
a) Numerical stability of least-squares estimation
$\pm 0.02$
b) Accuracy of consistency/model tests
$\pm 0.13$
c) Mismodeling of maneuvers
$\pm 0.01$
d) Mismodeling of the solar corona
$\pm 0.02$
e) Annual/diurnal terms
$\pm 0.32$

the measurement of accelerations that could otherwise be below any offset imposed by thruster leaks, mismodeled maneuvers, etc.. In order to stay clear from the radio beam, and also to provide shielding from solar radiation and wind, this sphere is ejected towards the "front" of the primary craft; in order to provide for the smoothest release possible, its drift speed should be relatively low, say $1 \mathrm{~mm} / \mathrm{s}$ to $1 \mathrm{~cm} / \mathrm{s}$. Furthermore, as in the case of the Pioneer probes, the primary craft is not three-axis stabilized, but instead spins around its axis of inertia: this allows for an averaging of off-axis systematic effects and provides a "cleaner" signal than would be available from the latter stabilization method, which would require a careful modeling of micro-thrust maneuvers and effectively reduce the measurement sensitivity (this should be the reason why the Pioneer anomaly has only been detected on spin-stabilized probes - the Galileo and Ulysses missions, aside from the two homonymous explorers).

We remark that this proposal, although ideally implemented as a dedicated mission, could also be deployed as a "piggy-back" payload onboard another deep space probe; this would help to circumvent funding constraints, reduce overall costs and strengthen the scientific scope of both missions. Clearly, the trade-off is a decrease in tracking precision of the primary craft, since it would encompass other scientific instruments and more complex design, increasing the noise level of both the Doppler tracking and the onboard acceleration measurements.

We now ascertain the magnitude of the various components present in Eq. (1), regarding this passive sphere. We 
do this by scaling the upper bounds presented in Table I (a more rigorous, but less intuitive description of the various components can be found elsewhere [9]; all results are mutually compatible). We assume a radius of $10 \mathrm{~cm}$ and a mass of $1 \mathrm{~kg}$ for the sphere, and a radius of $1 \mathrm{~m}$ and mass of $200 \mathrm{~kg}$ for the primary craft. With these quantities, we may scale the relevant acceleration components and obtain an order of magnitude budget of the systematic effects affecting the passive sphere:

i) Solar radiation and pressure: we assume the thermal emissivity of the sphere to be of the same order of magnitude or lower than that of the primary craft; given the area dependence, we obtain

$$
a_{\text {Pres.sphere }}=\left(\frac{r_{\text {sphere }}}{r_{\text {craft }}}\right)^{2} \frac{m_{\text {craft }}}{m_{\text {sphere }}} a_{\text {Pres.craft }}=2 a_{\text {Pres.craft }}<6 \times 10^{-12} \mathrm{~m} / \mathrm{s}^{2} .
$$

Hence, this component cannot be mistaken as an anomalous acceleration with magnitude $a_{P}=8 \times 10^{-10} \mathrm{~m} / \mathrm{s}^{2}$. Furthermore, one could exploit the advantageous cover (but not essential, given the low value of $a_{P r e s . s p h e r e}$ ) that the primary craft provides: the sphere is effectively shielded from these acceleration components by the "shadow cone" of the primary craft, up to a distance $d$ given by the inequality $d / r_{\text {craft }}=r / R_{\odot}$, where $r$ is the distance from the primary craft to the Sun and $R_{\odot}$ the radius of the Sun, from which we get $d \approx 100(r / 1 A U)$. Assuming a minimum clear distance (that is, sufficiently far away from the primary craft) of $50 \mathrm{~m}$, we conclude that the solar radiation and pressure are blocked by the primary craft after it has achieved a distance from the Sun of $5 A U$, close to Jupiter's orbit. Recall that the solar pressure affecting the primary craft falls below the reported magnitude of the Pioneer anomaly at $r \approx 4-10 A U$ : a mission profile would possibly encompass a low-power initial phase before this distance is achieved, with calibration and other tests performed - followed by a second phase, in which high-precision measurements would be conducted.

ii) Drag force: this force is proportional to the square of the velocity of the sphere with respect to the surrounding medium, and to its cross-section; given that the drift speed from the primary craft is very low compared to the latter's speed, one obtains the same scaling law as in the above case and, therefore, one can exclude drag forces as a competing effect with the anomalous acceleration under scrutiny.

iii) Electromagnetic forces: here we address the Lorentz forces due to interaction with cosmic rays, planetary and solar magnetic fields and charged plasma in the vicinity of the probe; these are proportional to the charge of the sphere, and also to its speed, which is approximated by that of the primary craft. Considering any electric charge to be evenly distributed throughout the volume of the probe, prior to the ejection of the sphere, we obtain the scaling law

$$
a_{\text {EMsphere }}=\left(r_{\text {sphere }} / r_{\text {craft }}\right)^{3}\left(m_{\text {craft }} / m_{\text {sphere }}\right) a_{\text {Lorentzcraft }}=0.2 a_{\text {Lorentzcraft }} \leq 10^{-15} \mathrm{~m} / \mathrm{s}^{2} ;
$$

this indicates that electromagnetic forces affecting the sphere may be neglected.

iv) Electrostatic force due to primary: assuming that the probe is charged with a total charge $Q$, evenly distributed, one may estimate the charge of the passive sphere as $Q_{\text {sphere }} \approx\left(r_{\text {sphere }} / r_{\text {craft }}\right)^{3} Q_{\text {craft }} \approx\left(r_{\text {sphere }} / r_{\text {craft }}\right)^{3} Q$. Hence, the electrostatic force between the passive sphere and the primary craft, at a distance d, is given by

$$
F_{\text {Coulomb }}=\frac{1}{4 \pi \epsilon_{0}}\left(\frac{r_{\text {sphere }}}{r_{\text {craft }}}\right)^{3}\left(\frac{Q}{d}\right)^{2} .
$$

Charge measurements on spacecraft are usually referred by the equivalent induced voltage $U$; if we simplistically assume a spherical body of radius $r$, one gets $U=Q / 4 \pi \epsilon_{0} r$. Hence, the above simplifies to

$$
a_{\text {Coulomb }}=\frac{F_{\text {Coulomb }}}{m_{\text {sphere }}}=4 \pi \epsilon_{0} \frac{r_{\text {sphere }}^{3}}{m_{\text {sphere }} r_{\text {craft }} d^{2}} U^{2} \approx 1.1 \times 10^{-13}\left(\frac{U}{1 V}\right)^{2}\left(\frac{d}{1 m}\right)^{-2} \mathrm{~m} / \mathrm{s}^{2} \text {. }
$$

Considering a minimum distance of $50 \mathrm{~m}$ shows that the acceleration of the passive sphere due to the Coulomb force is smaller than $10^{-12} \mathrm{~m} . \mathrm{s}^{-2}$ for a primary craft's potential $U$ below $23 \mathrm{kVolt}$, and smaller than the reported magnitude of the Pioneer anomaly below a potential as large as $19 \mathrm{MV}$ olt. Notice that the primary craft is also attracted towards the passive sphere; however, since this acceleration is much smaller than the inverse (by a factor $\left.m_{\text {sphere }} / m_{\text {craft }}=200\right)$, the relative acceleration is well approximated by the above. The first obtained limit, although extremely high, may sometimes occur in space environs, particularly during nightside magnetospheric storms [10], where the potential can reach approximately $20 \mathrm{kVolt}$. 
From the above, some guidelines for controlling the effect of Coulomb forces may be sketched: firstly, to guarantee that the ejection of the passive sphere occurs at a mild magnetic environment, in the absence of increased solar activity and away from magnetic belts of planets and other sources. Also, the probe should encompass a device to measure its own electric potential. Furthermore, instead of an overall control of the probe's charge, one may simply control the charge deposited in the passive sphere. Indeed, the above reasoning assumed an evenly distributed (volume) charge; even without charge balancing of the primary craft, any reduction of the sphere's charge would amount to an equal reduction in the magnitude of the related acceleration.

Although one lacks the proper knowledge to fully address this issue, two mechanisms may be outlined to diminish the charge deposition in the passive sphere; firstly, field-emission cathodes may be used to electrically neutralize it, in a similar fashion to the method used to control the gyroscopes of the Gravity Probe B [11]. Secondly, the passive sphere may be discharged by contact, prior to release, by establishing a parallel circuit with a capacitor with a much larger capacity; assuming that the passive sphere has a dielectric constant of approximately $\epsilon_{r}=3.75$ (value for a corner cube with fused silica), its capacity is $C=4 \pi \epsilon_{0} \epsilon_{r} r_{\text {sphere }} \approx 20 \mathrm{pF}$; a capacitor of just $1 \mathrm{nF}$ would diminish the sphere's charge by a factor of $98 \%$.

v) Thermal radiation from the primary craft: given that the radio beam points oppositely to the ejected sphere, one should only account for thermal radiation arising from the reflected emission of the RTGs and cooling of the primary craft; following Ref. [1], we assume that the dissipated power from the main hub has an upper bound of order $100 \mathrm{~W}$; also, we assume that the RTGs emit approximately tenfold. However, the design of a dedicated probe should minimize this, by concealing the RTGs into the geometry of the craft; hence, we assume that only a fraction of $10 \%$ (an extremely generous estimative) is reflected onto the sphere. Assuming isotropy, the sphere is subjected to an incident power given by $P=110 \mathrm{~W}\left(r_{\text {sphere }} / d\right)^{2}$; for distances larger than $500 \mathrm{~m}$, this is smaller than $1.1 \mu W$. The ensuing acceleration is then $a_{\text {thermal }}=P / m_{\text {sphere }} c \leq 3.7 \times 10^{-15} \mathrm{~m} / \mathrm{s}^{2}$, and can thus be neglected.

vi) Recoil from the laser ranging: given the above paragraph, a laser ranging unit with power of the order of $1 \mathrm{~mW}$ directly focused at the passive sphere imposes an acceleration of $a_{\text {laser }}=P / m_{\text {sphere }} c \leq 3.3 \times 10^{-12} \mathrm{~m} / \mathrm{s}^{2}$; this is possibly within the accuracy of the measurement, but clearly below the necessary magnitude to compete with the anomalous acceleration.

From the above, we conclude that all environmental and known systematics may be factored out of the problem, so that one must only be concerned with unaccounted systematics and forces manifesting new physics, regarding both the passive sphere and the primary craft. Hence, the relative acceleration between them is given by

$$
\mathbf{a}_{R} \equiv \mathbf{a}_{\text {craft }}-\mathbf{a}_{\text {sphere }}=\frac{\mathbf{F}_{\text {Syst.craft }}}{m_{\text {craft }}}+\frac{\mathbf{F}_{\text {Unk.craft }}}{m_{\text {craft }}}-\frac{\mathbf{F}_{\text {Unk.sphere }}}{m_{\text {sphere }}}
$$

where $\mathbf{F}_{\text {Syst.craft }}$ refers to possible, unaccounted systematic effects that may account for the Pioneer anomaly.

If the Pioneer anomaly is a real effect, imposing an equal acceleration upon bodies of different mass or composition, then the $\mathbf{F}_{U n k}$ terms should cancel, and $\mathbf{a}_{R}=\mathbf{F}_{\text {Syst.craft }} / m_{\text {craft }}$ : this allows for a direct discrimination of the systematic effects affecting the primary craft, which could offer a solution for the Pioneer anomaly without the need of new physics.

\section{PHYSICALLY ANOMALOUS DOPPLER TRACKING}

Asides from a magnitude of $a_{P}=(8.74 \pm 1.33) \times 10^{-10} \mathrm{~m} / \mathrm{s}^{2}$, the reported anomalous acceleration is widely credited as an attractive, sunbound effect. However, the determination of the direction of the anomaly is troublesome, given that the angular precision of the Pioneer probes was insufficient to distinguish between the different possibilities: a sunbound physical acceleration, an Earthbound effect due to misunderstood Doppler tracking or some other effect, an axis of inertia directed acceleration, reflecting some overlooked engineering or thermal effect, etc. A thorough analysis of the earlier stages of the Pioneer missions may clarify these issues, and the increased precision of a dedicated probe would certainly work towards that objective.

There is, however, a resilient ambiguity concerning the determination of the direction of the anomaly. Indeed, if it is found that the anomalous acceleration points towards the Earth, thus excluding a potential gravitational origin, one question remains: is it due to some unaccounted effect due to the power radiated from the aligned antenna (or other equipment, given that any thermal radiation along the axis of inertia should be aligned with it? Or is the anomaly due to a poorly understood Doppler effect? In fact, the latter issue is more general, since the current sunbound direction is also extrapolated from Doppler tracking. Here, the question is not if the trajectory was correctly analyzed and compared with ephemerides models, but if the actual underlying physical mechanism behind the tracking, the Doppler effect, is properly interpreted. This is a commonly neglected concern in the available literature, which tends to allow only for new physics of gravitational origin, with the Sun as source; however, an anomalous Doppler effect 
would still be of great theoretical interest, hinting further towards a better understanding of General Relativity and, possibly, future extensions and competing theories.

Given that the laser ranging used for tracking of the passive sphere relies on the "bounce" time of the emitted pulses, it should not be affected by any anomalous Doppler shift. Hence, if this is the origin of the Pioneer anomaly, it should not be probed by the measurement of the relative acceleration between the passive sphere and the primary craft:, the $\mathbf{F}_{\text {Unksphere }}$ term should vanish, while the $\mathbf{F}_{\text {Unkcraft }}$ should subside, instead of canceling each other in the relative acceleration. In order to strengthen the test of this hypothesis, we believe that an independent low frequency accelerometer should be a priority payload of the primary craft. If this is the case, not only will an anomalous Doppler shift be shown by the measurement of the passive sphere's relative acceleration, but the difference between measurements of the acceleration of the primary craft through Earth-based Doppler tracking and the onboard accelerometer would also reveal this effect. Notice, however, that an accelerometer will show no anomalous signal if the Pioneer anomaly is of gravitational origin, since both the spacecraft and the device's test mass will be subject to the same acceleration.

\section{BEYOND THE PIONEER ANOMALY}

Although the proposed dedicated mission to test the Pioneer anomaly has been viewed from this specific point of view, it can be more generally regarded as a probe to measure the acceleration profile of the Solar System. In this sense, one can speak of a scientific objective beyond the Pioneer anomaly: the accurate scrutiny of very low magnitude effects in widely different environs of the solar system. We now address some of the possibilities that such an instrument may offer us.

\section{A. A Yukawa force}

As was noted early on [1], a possible interpretation of the Pioneer anomaly is that it is due to an added Yukawa force, possibly due to to the dynamics of a massive scalar/vector field with the Sun as source. The full gravitational potential may then be written as

$$
V(r)=-\frac{G M_{\odot}}{(1+\alpha) r}\left(1+\alpha e^{-r / \lambda}\right)
$$

where $\alpha$ is the coupling strength and $\lambda$ is the range of the Yukawa component, inversely proportional to the second derivative of the scalar potential affecting the dynamics of the field, taken at its vacuum expectation value. An expansion of the derived acceleration $a=-d V / d r$ shows that, asides from the usual inverse square law Newtonian term, one obtains a constant term which may be identified with the Pioneer anomalous acceleration, $a_{P}=-a_{N} \alpha[2(1+\alpha)]^{-1}(1 A U / \lambda)^{2}$, where $a_{N}=G M_{\odot} /(1 A U)^{2}=5.93 \times 10^{-3} m . s^{-2}$ is the Newtonian acceleration at the distance of $1 A U$. This identification yields the curve $\alpha(1+\alpha)^{-1}(1 A U / \lambda)^{2}=-2 a_{P} / a_{N}=-2.95 \times 10^{-7}$; one remarkable feature is the negative coupling strength, which is characteristic of massive vector fields.

Typically, one chooses the lengthscale of the Yukawa force $\lambda$, and derives its strength from the solution curve, so that $\alpha \ll 1$. However, little attention has been paid to the remaining terms of the Taylor expansion; these can be written as $a_{n}=C_{n} a_{P}(r / \lambda)^{n}$, with $n=1,2, \ldots$ and $C_{n}<1$. Assuming $\lambda>r$ in the region of interest, the next to leading order is the term $a_{1}=(2 / 3) a_{P}(r / \lambda)$; for distances between $R_{i}=20 A U$ and $R_{o}=67 A U$, this linear term must be smaller than the allowed error margin for the reported anomalous acceleration, which amounts to $15.2 \%$ of its center value. Thus, we obtain

$$
\frac{2}{3} \frac{a_{P}}{\lambda} \leq \frac{2 \times 0.152 a_{P}}{R_{o}-R_{i}}
$$

which implies that $\lambda \geq 2.19\left(R_{o}-R_{i}\right) \simeq 103 A U$.

\section{B. A probe of the Kuiper belt mass distribution}

As shown in Ref. [7, 8], the mass distribution of the Kuiper belt cannot account for the Pioneer anomaly, providing neither its magnitude nor its constant behaviour (or allowed spatial variation [12]). However, the acceleration sensitivity of the discussed dedicated probe could, in principle, be of great use in discriminating between different models 


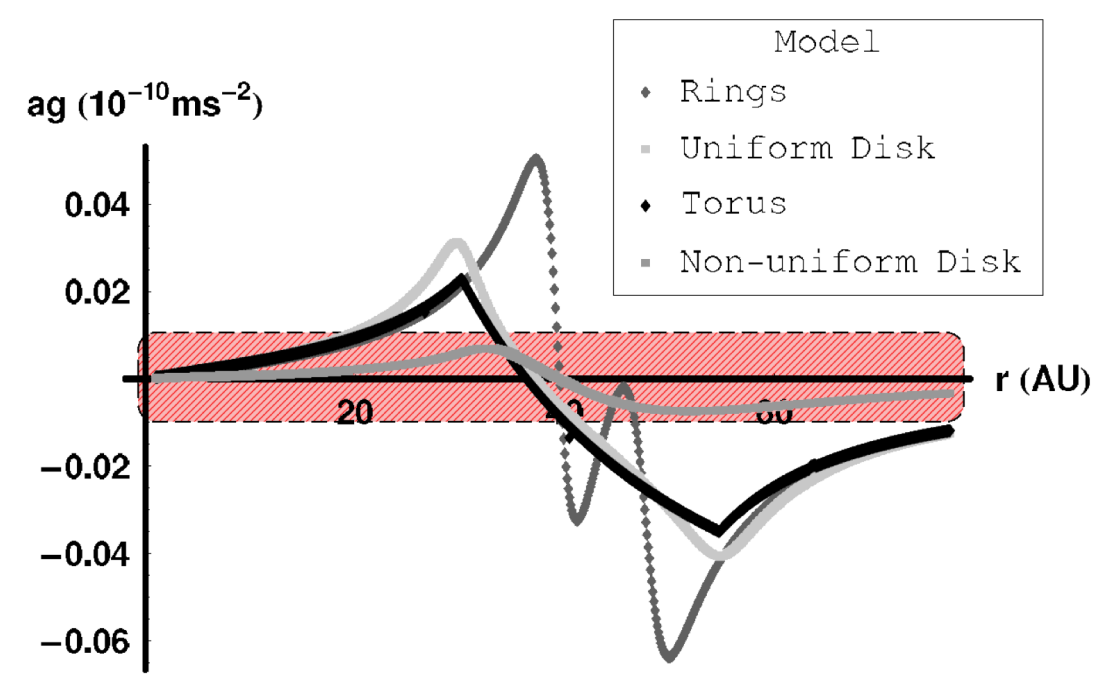

FIG. 1: Kuiper belt mass distributions, from Ref. [7], with an accuracy threshold of $10^{-12} \mathrm{~m} / \mathrm{s}^{2}$ superimposed.

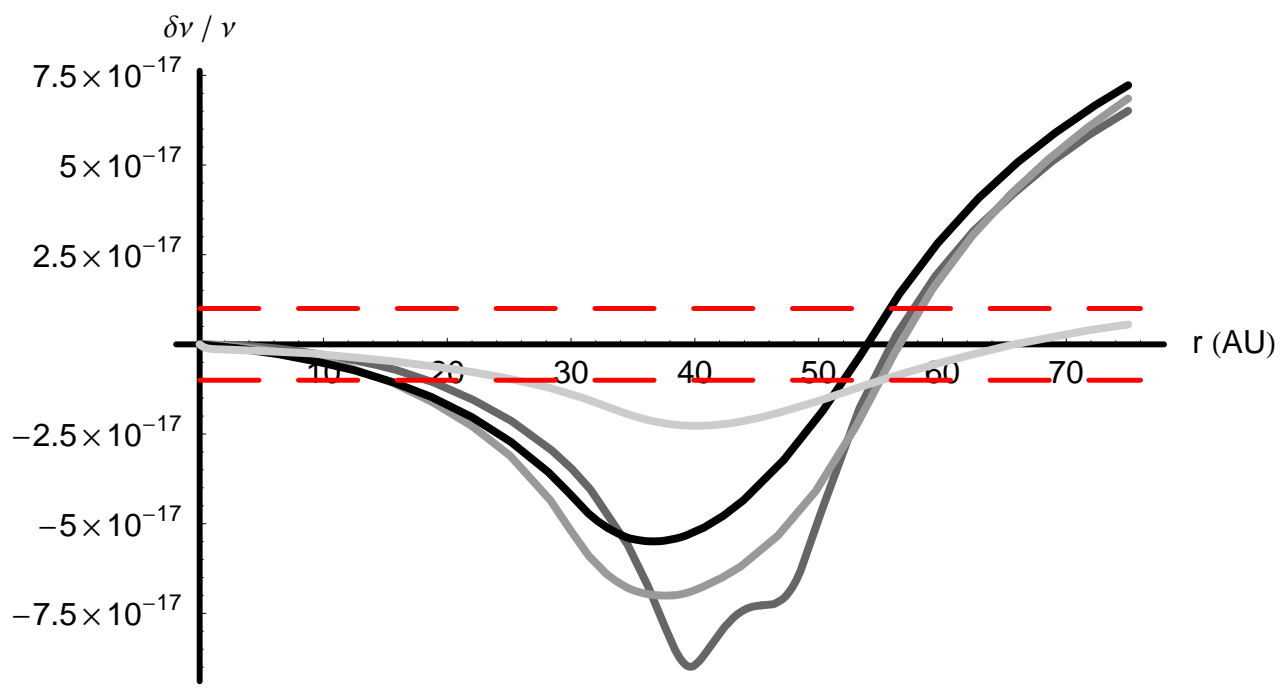

FIG. 2: Relative frequency shift due to different Kuiper Belt mass distributions for a solar ecliptic latitude of $\theta=3^{\circ}$, with an accuracy threshold of $10^{-17}$ superimposed (same labels as in Fig. 1).

for the spatial distribution of the estimated 0.3 $M_{\text {Earth }}$ Kuiper belt. Indeed, by plotting the different acceleration profiles of competing models (taken from Ref. [7]), against the assumed $10^{-12} \mathrm{~m} / \mathrm{s}^{2}$ accuracy in Fig. 1, one concludes that the former should be detectable for a wide range. Specifically, one gets the following "positive signature" intervals, where gravity from the Kuiper belt could be measured with the onboard accelerometer: for the two rings mass distribution, this starts at $23 A U$, with gaps at $40 A U$ and $41-47 A U$; for the uniform disk mass distribution, this starts at $22 A U$, with a gap at $34-43 A U$; for the toroidal mass distribution, this starts at $22.5 A U$, with a gap at $33-42 A U$; finally, the non-uniform disk model is outside the limit of measurability, although its acceleration peaks at 32 and $48 A U$ could perhaps be detected by a careful analysis, particularly if the total mass of the Kuiper belt is slightly higher than acknowledged.

Alternatively, one could rely on precise Doppler tracking and, by measuring relative frequency shifts, directly probe 
the gravitational potential $U=c^{2} \Delta \nu / \nu$. By plotting the gravitational potential derived from different models for the Kuiper belt's mass distribution against an assumed accuracy of $10^{-17}$ in Fig. 2, we obtain the following positive signature intervals: the two rings mass distribution is detectable at the interval $18 A U<r<54 A U$ and for $r>58 A U$; the uniform disk mass distribution is detectable at the interval $15 A U<r<55 A U$ and for $r>58 A U$; the toroidal mass distribution can be detected at the interval $15 A U<r<52 A U$ and for $r>56 A U$; finally, the non-uniform disk can only be measured at $26 A U<r<55 A U$. Notice that, with the exception of the non-uniform disk mass distribution, all remaining models can be essentially detected from distances beyond $15 \mathrm{AU}$.

\section{CONCLUSIONS}

Recent years have witnessed an increasing interest concerning the Pioneer anomaly, elected by some as one of the withstanding enigmas of contemporary physics. This increased exposure has prompted for various proposals, of varying pertinence and scope. Asides from valid, although mainly phenomenological theories, some have hailed that the measured constant acceleration may be a manifestation of Modified Newtonian Dynamics (MOND) [13] in the Newtonian regime, or a signal of perturbative corrections to the geodetic motion of test particles, due to the presence of a cosmological constant; although this was not the purpose of this study, the authors cannot refrain from stating that, in our view, such claims stem solely from fortuitous numerical coincidences.

Indeed, in the former case, the observed anomalous acceleration is of the same order of magnitude (within a factor of eight) as the fundamental acceleration cutoff of MOND, $a_{P} \sim a_{0} \approx 10^{-10} \mathrm{~m} / \mathrm{s}^{2}$; however, this similitude is not supported by any available study of the Newtonian regime of neither the phenomenological MOND model, nor Tensor-Scalar-Vector theory (TeVeS), its backing fundamental framework. Such an unsubstantiated claim appears recursively in specialized and general discussions and media, but the lack of proof or argument serves only to discredit it as a viable modification or extension of General Relativity. Also relevant, this suggestion is raised at a particularly troublesome time for MOND and TeVeS alike, when the existence of Dark Matter is being directly probed in galaxy clusters [14], and the viability of these proposals is at stake, if not yet doomed $[15,16]$.

In the latter case, the identification of the Pioneer anomalous acceleration as an effect induced by the expansion of the Universe is doubly mischievous; firstly, most approaches rely on the apparent relation $a_{P} \sim c H$, and attempt to derive this expression from first principles, often with misguided arguments; indeed, the correct use of the Schwarzschild-de Sitter metric, which extends the standard, static and spherically symmetric metric, matching it with a de Sitter boundary, yields an acceleration which is lower than the reported value by over eleven orders of magnitude! Furthermore, and perhaps more concerning, most of these claims fail to account for the simple problem with this identification: an acceleration due to the expansion of the Universe is, by nature, repulsive, while the anomalous acceleration of the Pioneer probes is sunbound.

This said, one should exert this criticism with caution; indeed, while the above proposals do not seem at all adequate, an immediate dismissal of other, more evolved models could inhibit the development of a phenomenologically viable extension of General Relativity, and perhaps hinder a better understanding of gravitation [17]. Indeed, a confirmation of the existence of an anomalous, constant acceleration in the Solar System could signal the beginning of a new cycle in the pursuit for a clearer grasp of the surrounding Universe. But, for now, one must proceed with care, and further examine all possible "natural" causes for the phenomena, with the before-mentioned modeling of thermal effects as a prime candidate for inspection. Even if this comes out as the definitive explanation for the anomaly, a highly valuable service will be done for the scientific community, allowing for better deep space tracking, more accurate ephemerides computation, and improved spacecraft design.

In either case, one thing is clear: prospects of new physics, any mismodelling or engineering cause, the road towards a better understanding of the Pioneer anomaly lies still ahead. 


\section{Acknowledgments}

This work is partially supported by the Programa Dinamizador de Ciência e Tecnologia do Espaço of the FCT - Fundação para a Ciência e Tecnologia (Portuguese Agency), under the project PDCTE/FNU/50415/2003, and partially written while attending the second Pioneer Anomaly Group Meeting at the International Space Science Institute (ISSI) at Bern, from 19 to 23 of February 2007. The authors would like to thank ISSI and its staff, for hosting the group's meeting and accommodating for logistic requirements. The work of JP is sponsored by the FCT under the grant BPD 23287/2005.

[1] J. Anderson, P. Laing, E. Lau, A. Liu, M. Nieto and S. Turyshev, Phys. Rev. Lett. 81 (1998) 2858; Phys. Rev. D65 (2002) 082004 .

[2] L. Scheffer, Phys. Rev. D67 (2003) 084021.

[3] Pioneer Anomaly Collaboration, European Space Agency Cosmic Vision: Space Science for Europe 2015 - 2025, BR-247, gr-gc/0506139.

[4] O. Bertolami and J. Páramos, Class. Quantum Gravity 21 (2004) 3309.

[5] G. Null, Ap. J. 81 (1976) 1153.

[6] I. Mann and H. Kimura, J. Geophys. Res. A105 (2000) 10317.

[7] O. Bertolami and P. Vieira, Class. Quantum Gravity 23 (2006) 4625.

[8] M. Nieto, Phys. Rev. D72 (2005) 083004.

[9] K. Penanen and T. Chui, Nuclear Physics Proceedings Supplement 134 (2004) 211.

[10] R. Grard, K. Knott and Pedersen, Space Science Reviews 34 (1983) 289.

[11] S. Buchman et al., Advances in Space Research 25 (2000) 1181.

[12] C. Markwardt, gr-qc/0208046.

[13] M. Milgrom, Ap. J. 270 (1983) 365; J. Bekenstein, Phys. Rev. D70 (2004) 083509.

[14] D. Clowe et al., to appear in Ap. J., astro-ph/0608407; R. Gavazzi et al., Astron. Astrophys. 403 (2003) 11.

[15] O. Bertolami and J. Páramos, gr-qc/0611025.

[16] R. Takahashi and T. Chiba, astro-ph/0701365.

[17] O. Bertolami, to appear in Int. J. Mod. Phys. D , astro-ph/0608276. 